\title{
A method for testing synchronization to a musical beat in domestic horses (Equus ferus caballus)
}

\author{
MICAH R. BREGMAN \\ Department of Cognitive Science, UC San Diego \\ and The Neurosciences Institute \\ JOHN R. IVERSEN \\ The Neurosciences Institute, \\ and Swartz Center for Computational Neuroscience, UC San Diego \\ DAVID LICHMAN \\ Parelli Natural Horsemanship, 5-Star Professional \\ MEREDITH REINHART \\ Private Scholar \\ ANIRUDDH D. PATEL [1] \\ The Neurosciences Institute \\ and Department of Psychology, Tufts University
}

\begin{abstract}
According to the "vocal learning and rhythmic synchronization hypothesis" (Patel, 2006), only species capable of complex vocal learning, such as humans and parrots, have the capacity to synchronize their movements to a musical beat. While empirical research to date on a few species (e.g., parrots and monkeys) has supported this hypothesis, many species remain to be examined. Domestic horses are particularly important to study, as they are vocal non-learners who are occasionally reported to move in synchrony with a musical beat, based on informal observations. If these reports are substantiated by scientific experiments, this would challenge the vocal learning hypothesis and provide a new species for the comparative study of musical rhythm. Here we present a new method for testing whether horses can synchronize their trotting to a musical beat, including an illustration of data analysis based on data collected from one horse.
\end{abstract}

Submitted 2012 July 30; accepted 2012 September 6.

KEYWORDS: musical beat, rhythm, synchronization, animals, horses, evolution

\section{INTRODUCTION}

THIS paper presents a method for testing synchronization of movement to a musical beat in domestic horses (Equus ferus caballus). As discussed below, horses provide a crucial test of the "vocal learning and rhythmic synchronization hypothesis" (Patel, 2006), which states that the capacity for complex vocal learning is an evolutionary and neurobiological prerequisite for the ability to synchronize movements with a musical beat. One prediction of this hypothesis is that vocal non-learners (such as nonhuman primates, dogs, cats, and horses) lack the capacity to synchronize movements to a musical beat. Among vocal nonlearners, domestic horses are of particular interest because unlike dogs, cats, and nonhuman primates, they are the subject of numerous anecdotes about spontaneous entrainment to a musical beat (one such anecdote is reproduced in Appendix 1; author AP has received several such anecdotes from equestrians). If these anecdotes can be confirmed by scientific experiments, this would provide evidence contrary to the vocal learning hypothesis and provide a new species for the comparative study of musical rhythm. Hence the current paper presents a method for testing synchronization to a musical beat in horses, including illustrations of data analysis based on data collected from one horse. It should be noted that these data are not sufficient in quantity to determine if horses synchronize to music, and are presented here for didactic purposes. The focus of the paper is on describing a new experimental method, called "circular trotting to 
music" (CTM), with the hope that future researchers will use it to decisively answer whether horses synchronize to the beat of music. Before describing the CTM method, we first briefly discuss the theoretical background for this research and review several key features of musical beat perception and synchronization.

\section{Theoretical Background}

Why study music perception in other animals? Cross-species studies of music cognition provide a way to study the evolutionary roots of our musical capacities (McDermott \& Hauser, 2005; Fitch, 2006). Music cognition involves many distinct capacities, ranging from "low-level" capacities not specific to music, such as the ability to perceive the pitch of a complex harmonic sound, to "high-level" capacities which appear unique to music, such as the processing of tonal-harmonic relations based on learned structural norms (Peretz \& Coltheart, 2003; Koelsch, 2011). It is unlikely that all of these capacities arose at the same time in evolution. Instead, different capacities are likely to have different evolutionary histories. Cross-species studies can help illuminate these histories using the methods of comparative evolutionary biology (for further discussion, see Patel \& Demorest, 2013).

For example, consider the capacity to sense, and move in synchrony with, a perceived regular temporal pulse (e.g., via foot tapping, head bobbing, or dance). Musical beat perception and synchronization (BPS) is a fundamental aspect of music cognition that develops without special training in young children, and is observed in every human culture (Brown \& Jordania, 2011; Nettl, 2000). Did the capacity for BPS arise uniquely in the human lineage as part of an evolutionary adaptation for musicmaking (Bispham, 2006; Honing, 2012), or does it reflect ancient abilities shared by many species? Darwin (1871) seems to have favored the latter view, stating that "The perception, if not the enjoyment, of musical cadences and of rhythm is probably common to all animals, and no doubt depends on the common physiological nature of their nervous systems" (p. 1207 in the 2006 edition, E.O. Wilson, Ed.). Darwin's intuition is reasonable: it seems plausible that the capacity to sense a beat in music and synchronize to it could be widespread among animals. After all, the auditory systems of humans and other mammals (especially primates) have many structural and functional parallels (Rauschecker \& Scott, 2009), and all vertebrates produce voluntary rhythmic movements (e.g., as part of locomotion). Therefore, it is reasonable to expect that many animals could learn to coordinate rhythmic movements with a perceived auditory beat. Furthermore, nonhuman animals (henceforth, animals), such as rhesus monkeys, can learn complex sensorimotor tasks in the laboratory (Georgopoulos, Taira, \& Lukashin, 1993). Thus one might expect that many animals could learn to synchronize movements with an auditory beat, especially since rhythm is increasingly thought to be a fundamental organizing principle of brain function (Buzsáki, 2006).

Another conceptual possibility is that the capacity for BPS is neither uniquely human nor widespread among animals, but restricted to a few species. This view was suggested by Patel (2006), who proposed that the capacity for BPS arose as a fortuitous byproduct of the brain circuitry for complex vocal learning. According to this view, the capacity for BPS did not arise as a result of an evolutionary adaptation for music-making, but a secondary use (or "exaptation", Gould \& Vrba, 1982) of brain circuits that evolved for other reasons. This "vocal learning and rhythmic synchronization hypothesis" was motivated by the fact that vocal mimicry, like BPS, involves tight links between the auditory and motor systems, and the fact that the neural substrates for vocal learning and BPS appear to overlap in the brain (see Patel, 2006 for details). Crucially, vocal learning is a rare capacity found only in a few groups of mammals (including humans, dolphins, elephants, and seals) and birds (songbirds, parrots, and hummingbirds), and is associated with neuroanatomical specializations including modified auditory-motor cortical connections (Jarvis, 2007). Thus the vocal learning hypothesis makes a testable prediction: only vocal learners are capable of BPS.

The discovery of BPS in parrots has supported this hypothesis (Hasegawa, Okanoya, Hasegawa, \& Seki 2011; Patel, Iversen, Bregman, \& Schulz 2009a; Schachner, Brady, Pepperberg \& M. Hauser, 2009) as has the finding that rhesus monkeys (who are vocal non-learners) could not learn to synchronize their taps to a metronome despite over a year of concerted training (Zarco, Merchant, Prado, \& Mendez, 2009). However, many other vocal non-learner species remain to be tested. As noted above, horses are of particular interest due to the many anecdotal accounts of BPS in domesticated horses. If horses show the key features of BPS, this would challenge the vocal learning hypothesis and raise new questions about the evolutionary substrates of this ability. 


\section{Key features of musical beat perception and synchronization}

Synchronization to a musical beat in humans has several important features that distinguish it from other examples of rhythmic entrainment in nature, such as the synchronous chorusing of certain insects and frogs (Patel et al., 2009b). First, BPS involves the extraction of a beat from a complex acoustic stimulus (i.e., music vs. from simple pulse trains). Second, BPS involves substantial flexibility in movement tempo: humans can easily synchronize their movements to a musical beat across a fairly wide range of tempi. Third, BPS is cross-modal, with rhythmic sound driving movement that is not necessarily aimed at sound production. Thus a convincing demonstration of BPS in animals requires demonstrating these three features. In addition, an important feature of BPS is phase matching: people spontaneously align their taps and other rhythmic movements with a beat. That is, people tap slightly before the beat or on the beat. Stated more formally, the average temporal asynchrony between taps and beats is slightly negative or around zero (Patel, Iversen, Chen, \& Repp, 2005; Rankin, Large, \& Fink, 2009). This indicates accurate temporal prediction of beats. Hence if an animal is trained to tap with a beat, and taps at the correct tempo after each beat (e.g., with a delay of a few hundred $\mathrm{ms}$ ), this behavior could be largely reactive rather than anticipatory. (This sort of behavior was observed in the study of Rhesus monkeys mentioned above.)

\section{PRIOR RESEARCH ON HORSES AND MUSIC}

To our knowledge, there is no prior research on synchronization of movement to music in horses. Indeed, there appears to be almost no scientific research on how horses respond to music. This is somewhat surprising given the frequent use of music in certain types of horse training and dressage (a competitive sport based on guiding a horse through a complex set of movements based on subtle cues from the rider). For example, the Spanish Riding School of Vienna, a traditional riding school for Lipizzan horses that dates back to the 1500s, has long used music in its performances. The belief that horses had "an almost human sensibility for music" was proposed as early as 1563 by French author François de Provane, and in 1612 the French court staged an elaborate equestrian ballet for the engagement of Louis XIII (Van Orden, 2005: 235-284).

The lack of modern research on equine responses to music is also somewhat surprising given that several other species have been studied for responses to human music, motivated by either basic cognitive research or applied animal science (e.g., McDermott \& Hauser, 2007; Patel et al., 2009; Schachner et al., 2009; Snowdon \& Teie, 2010; Wells, Graham, \& Hepper, 2002; Wells, 2009). We were able to find only one prior scientific paper on horses and music, which examined the effect of four types of music on stressrelated behaviors (such as neighing) in ponies (Houpt, Marrow, \& Seeliger, 2002). The authors found no significant differences between different music types.

Fortunately, there is excellent psychophysical research on horse hearing that is relevant to the current work. Figure 1 shows an audiogram for domesticated horses (solid line) compared to a human audiogram (dashed line) (Heffner \& Heffner, 1992). These audiograms represent the hearing thresholds for pure tones of different frequencies, i.e., the lowest intensity at which a pure tone can be detected $50 \%$ of the time. Larger threshold values indicate that the tone had to be more intense before it could be detected. Horses have a broad region of best hearing sensitivity, which overlaps considerably with the range of best hearing in humans. One notable difference is that the horse hearing range does not extend quite as low as the human range, but exceeds the human range on the high end. Heffner and Heffner (1992) suggest that high-frequency hearing in horses plays an important role in sound localization, rather than being used for acoustic communication with conspecifics (for one recent study of horse vocalizations, see Policht, Karadios \& Frynta, 2011). They also suggest that domestication of horses by humans, which has occurred for at least 5000 years, is unlikely to have significantly affected the horse audiogram.

Given the extensive overlap between horse and human audiograms, it seems likely that horses hear human music quite well. Spectral analysis of the two songs used in the current study indicated that the majority of the energy was below $2 \mathrm{KHz}$, which is typical for human music and well within the hearing range of both species. 


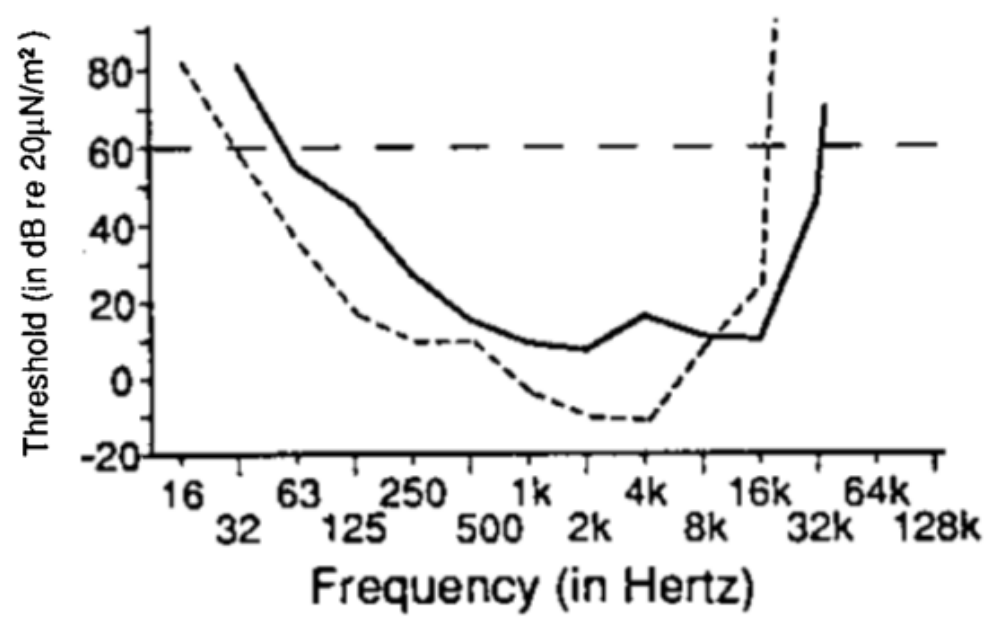

Fig 1. Audiogram of the domestic horse, Equus ferus caballus (solid line) compared to a human audiogram (dashed line). From Heffner \& Heffner (1992). The human audiogram was obtained from speakers placed in front of the subject, to make the testing conditions similar to animal testing. The horizontal line at $60 \mathrm{~dB}$ is conventionally used to define the low and high-frequency hearing limit, based on where it intersects the audiogram. Audiogram values below 0 on the y-axis indicate sounds whose intensity is less than the $0 \mathrm{db}$ reference level (i.e., $0 \mathrm{~dB}$ is not silence, but an established conventional sound pressure level). Reproduced with permission.

\section{TESTING MUSICAL BEAT PERCEPTION AND SYNCHRONIZATION IN HORSES}

\section{General considerations}

In testing animals for synchronization to a musical beat, several important methodological issues arise (see Patel et al., 2009b for a detailed discussion of 11 such issues). A primary issue is eliminating possible visual, auditory, or tactile rhythmic cues from humans, which could lead to a "Clever Hans" effect. This concern particularly applies to horses, which are capable of sensing human cues in many modalities (e.g., Proops et al., 2010; Saslow, 2002). In particular, the horse's sensitivity to tactile cues means that an essential requirement for studies of BPS is that the horse not be ridden. In addition, any humans in close proximity to a horse during testing should be "deaf" to the music that the horse is hearing so that they cannot give inadvertent cues.

Another primary issue is that the animal be tested for tempo flexibility, i.e., for its ability to synchronize to music at different tempi. The danger with using a single tempo is that a horse could appear to synchronize to music if the music tempo happened to match its natural trotting tempo, even without any auditory-motor coupling. In prior research on BPS in another species (a cockatoo), testing for tempo flexibility was accomplished by choosing a single song with a clear beat and creating versions at several different tempi using software that allows changing tempo without changing pitch (Patel et al., 2009a). In that prior work, 11 different versions of a song were created ranging from $20 \%$ slower to $20 \%$ faster than the original. The use of different tempi allows one to test for tempo flexibility, a key feature of BPS, and is also crucial for the statistical permutation tests used to check for true synchronization to a musical beat, as discussed further below. For certain animals, the number of tempi used might need to fewer, depending on the particular motor abilities of the animal. For example, with horses one should ensure that the horse is capable of trotting as slow as the slowest tempo and as fast as the fastest tempo. In general, we suggest that no fewer than five distinct tempi be tested (e.g., $-15 \%,-7.5 \%, 0,+7.5 \%$, and $+15 \%$ relative to the original musical piece). According to one source (Gallo, 2007), the average trotting tempo for horses is 152 beats per minute [BPM], based on the timing of footfalls of the front legs. That is, if one measures the number of steps per minute as the horse trots, counting each front leg footfall as a step, the average rate is 152 steps per minute, though this number depends on the particular horse. Author DL feels that the comfortable range of trotting for horses likely spans about $140-200$ BPM. Thus if using a tempo range of $+/-15 \%$ relative to the original musical piece, a base tempo of about 165 BPM (e.g. "Hound Dog" by Elvis Presley) would ensure that the slowest and fastest tempi fall within this range. 
A final issue is the use of objective methods to measure synchronization to a musical beat. Simply examining videos and making perceptual judgments of synchrony is not a valid way to study BPS in other species, as humans have a tendency to perceive synchrony between rhythmic auditory and visual stimuli even when it is not objectively present (Arrighi, Alais, \& Burr, 2006). Thus objective measures, such as frame-by-frame coding of the animal's movement from video (with the sound track turned off) are needed. Some portion (ideally all) of the data should be coded independently by at least two researchers in order to measure inter-rater reliability.

\section{The circular trotting to music (CTM) method}

The circular trotting to music (CTM) method is a way of testing whether horses spontaneously synchronize their trotting to a musical beat. This method involves a horse trainer and an equipment controller working with one horse in an indoor enclosure (to minimize background noise). The trainer stands at the center of the enclosure and the horse trots in circles while music is played over loudspeakers in the arena. To prevent the trainer from hearing this music, the trainer wears sound-isolating insert earphones and closed-ear noiseprotecting earmuffs (e.g., of the type used when operating loud power equipment). The earphones are connected to a portable digital audio device, and throughout the experiment the trainer listens to music without any beat, such as meditation music, at a sound level sufficiently high to mask out the music heard by the horse. The equipment controller triggers the start of experimental trials by starting the music and video camera, at which point $\mathrm{s} /$ he gives a visual cue to the trainer that an experimental trial has begun. Further information on the roles of the trainer and equipment controller are given below. Trials from the current study can be viewed online (see Appendix 2), and the reader is encouraged to watch these videos in conjunction with reading the information below. Our study focused on a young adult male horse named "True" (Breed: Morgan), who was approximately 6 years old. Testing took place in an indoor enclosure measuring approximately $75^{\prime}$ x 125', with a dusty sand floor (Horse Quarters in Davis, CA). The study took place in March of 2010.

\section{ROLE OF THE TRAINER}

The trainer interacts with the horse during the experiment. Specifically, once the music for a given trial has started, the trainer gives the horse a "go" signal to start trotting in circles around the trainer. The horse continues trotting until it elects to stop or the music ends (in which case the trainer gives a "stop" signal). The trainer can give the horse a food reward at the end of trials if needed.

In our study, the trainer and horse were connected by a long slack rope, a $22 \mathrm{ft}$ "lead line" (pronounced: "leed" line) made of yachting braid made by Parelli Natural Horsemanship, used in conjunction with a $1 / 4$ " yacht braid rope halter. The trainer held one end of this rope with one hand, and with the other hand held a thin rigid pole connected to a flexible whip (in Parelli terminology, a "carrot stick and string", Figure 2). Using body language, the trainer gave the "go" signal to the horse using the rope and/or whip. The horse was allowed to circle the trainer either clockwise or counterclockwise, depending on its preference, which could vary from trial to trial. As the horse circled (typically at a distance of about 10' from the trainer), the trainer turned in place to maintain eye contact with the horse. The trainer continued to hold the rope while the horse circled, but no tension was applied to the rope, which often dragged lightly on the ground, meaning that the horse set its own pace. The whip was never applied once the horse started trotting, and thus was not a source of rhythmic cues. On five trials in the current study the horse trotted around the trainer "at liberty", meaning that no rope connected the horse and trainer (the trainer still turned in place to face the horse as it trotted). Also, on three trials the horse started trotting before the music started playing. In future work, the use of a lead line (vs. liberty) and the onset of horse trotting relative to the music (before or after music onset) should be made consistent. 


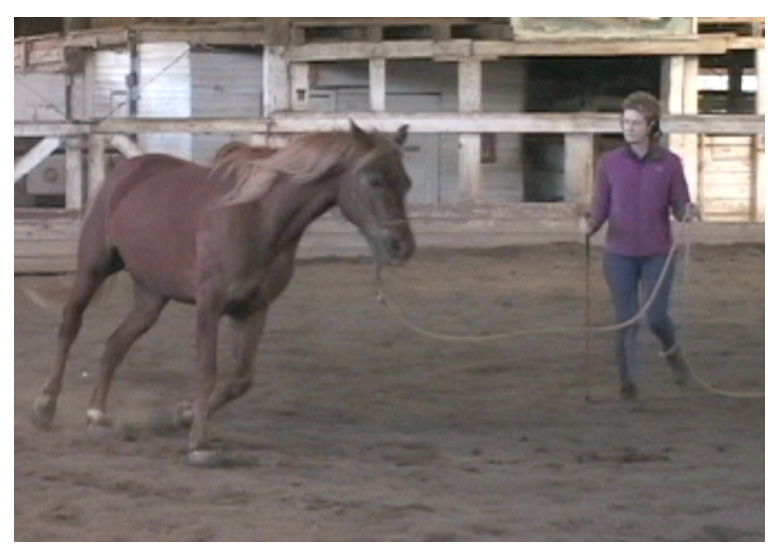

Fig. 2. Screenshot from video of an experimental trial, showing the horse and trainer. Please see the online videos for full experimental trials.

\section{ROLE OF THE EQUIPMENT CONTROLLER}

The equipment controller handles audio playback and video recording. S/he stands at a table some distance from the horse and trainer. The music is played over high-quality, powered portable speakers at a sound level that is clearly audible at the horse's position. The video camera, mounted on a tripod, is used to film all trials and should give a good view of both horse and trainer, with the camera angle set so that the horse's feet are clearly visible (since the timing of footfalls is used to measure synchronization of movement to the beat). Once the music and video camera have been triggered for a trial, the equipment controller should step behind a blind to ensure that $\mathrm{s} / \mathrm{he}$ is invisible to the horse.

In our study, music was presented via a JBL EON-10 powered speaker (280 Watts, dimensions 19" x 12" x 10", with 10 " woofer, 1 " tweeter, and frequency range of $58 \mathrm{~Hz}-18.5 \mathrm{kHz})$, connected to an iPod Classic (8 GB). Trials were filmed with a Canon Optura 20 digital video camera (frame rate $=30$ frames per second [fps]; to achieve higher-resolution analysis of animal movements, future work should use cameras with higher frame rates, e.g., 100 fps or more). The equipment controller and video camera were located about $50^{\prime}$ ' from the horse at the closest point of his trotting circle; the audio speaker was on a viewing platform on the side of the arena, 5' off the ground (about 15' from the equipment controller and $55^{\prime}$ from the True at the closest point of his trotting circle). We did not use a blind for the equipment controller, which raises the possibility that the horse may have picked up on inadvertent rhythmic cues from the controller, who heard the same music as the horse. Author DL (an experienced horse trainer who served as a trainer in the current work) feels it is unlikely that True sensed visual cues from the equipment controller, because True was focused on his trotting circle, and because the controller was out of view of True for at least $1 / 3$ of the circle while True was heading away from the controller. However, future work should use a blind to eliminate the possibility of cues from equipment controller to horse. An alternative to video coding might be to use an accelerometer attached to the horse, which has been used to capture human rhythmic movement to music (Phillips-Silver et al., 2011).

\section{CHOICE OF MUSIC AND NUMBER OF TRIALS}

Music that conveys a clear, steady beat should be selected for research on animal BPS. Much pop music is suitable for this purpose. It may be advantageous to use music familiar to the animal. For example, in the case of horses, a song that the horse has heard in its barn or during training or showing could be chosen. Care should be taken that the music does not sound muddy at the horse's position (e.g., due to poor quality audio speakers or echoes in the arena). As noted above, versions of the music at several different tempi should be presented. Furthermore, multiple "usable" trials should be gathered at each tempo, where a "usable" trial is a trial where the horse trots continuously for a sustained amount of time while the music is on (e.g., at least 50 steps). For example, if 5 distinct tempi are used, it would be desirable to have at least two or three useable trials at each tempo.

In our study two songs were used: "No one emotion" by George Benson (tempo $=155$ BPM, duration $=3: 58$ ) and "The Rhythm of the Ride" by Mary Ann Kennedy (tempo = 156 BPM, duration = 2:45; note that tempi reported here were obtained from the beat tracking algorithm discussed in the Data 
Analysis Method section below). These songs were chosen because they had a strong beat and because author DL, who had prior experience with True, felt that True could trot comfortably at these tempi.

Due to time constraints, we were not able to conduct trials with tempo-manipulated versions of the songs. Thus we cannot conduct the statistical permutation tests needed to determine if there is significant synchronization to the beat, although such tests are explained in the section on data analysis below. As noted at the beginning of this paper, these data are not sufficient to determine if True showed significant synchronization to a musical beat, but they are sufficient for an explanation of data analysis methods for testing BPS in horses. These methods are described below.

\section{Data analysis method}

The experimental video was segmented into individual trials based on the start and end of the music stimulus on each trial. For each trial, the video and audio tracks were separated so that an audio file and a silent video file were obtained for further analysis, using AviSynth (avisynth.org).

\section{AUDITORY BEAT EXTRACTION}

The auditory beat was extracted for each trial using a dynamic programming algorithm in Matlab (Ellis, 2007). Using this method, we obtained a sequence of music beat times for each of the experimental trials. To verify the accuracy of the beat tracker, we synthesized beeps representing each beat and overlaid them on top of the audio track. Any segments where the musical beat and beat tracker diverged were excluded from the analysis. In our data, one trial where beat tracking was poor was excluded.

\section{VISUAL RHYTHMIC MOVEMENT EXTRACTION}

The timing of the horse's left and right front footfalls was used as a measure of rhythmic movement. The timing of each footfall was extracted from the silent videos by two independent coders. For each segment, the coders recorded the frame number in which a footfall occurred, as defined by the point where either the left or right hoof made full contact with the ground (each footfall was labeled as a left or right footfall, but these labels were not used in the analysis: rather, the time sequence created by the alternation of the front two feet provided the movement series for analysis). Inter-rater reliability was very high: $90 \%$ of frame numbers recorded by coder 2 were within $1 / 15^{\text {th }}$ of a second of coder $1 ; 99 \%$ were within $1 / 10^{\text {th }}$ of a second), so one of the coder's data was used for all of the reported analyses. By converting frame numbers to time, we were able to obtain a sequence of footfall times (in seconds) for each segment. The total number of coded footfalls per trial ranged from 57 to $156($ mean $=101.0)$.

\section{STATISTICAL SYNCHRONIZATION ANALYSIS: IDENTIFYING SYNCHRONIZED BOUTS}

Following the methods of Patel et al. (2009), the statistical test of BPS is divided into two steps. The first step is to examine each trial for bouts of synchrony (i.e. time windows when footfalls and musical beats are aligned). This process was conducted on the current study, and is described in this subsection. The second step (not possible in the current study, due to lack of trials at different tempi) is a statistical permutation test that provides the true test of BPS. This permutation test is further described in the next subsection.

To look for synchronized bouts within a trial, the timing of each footfall was converted into a relative phase (RP) value within the time interval bounded by the preceding and succeeding musical beats (Figure 3). For example, if a musical beat occurred at 1.0 and 2.0 seconds and a footfall at 1.25 seconds, the footfall's RP would be coded as 90 degrees (1/4 of the beat interval after the beat); whereas a footfall at 1.50 seconds would be labeled as 180 degrees ( $1 / 2$ of the beat interval), and a footfall of 1.75 seconds would be coded as -90 degrees ( $1 / 4$ of the beat interval before the beat) Thus a sequence of $\mathrm{N}$ footfall times in a given trial would be converted to a sequence of $\mathrm{N} R P$ values. 


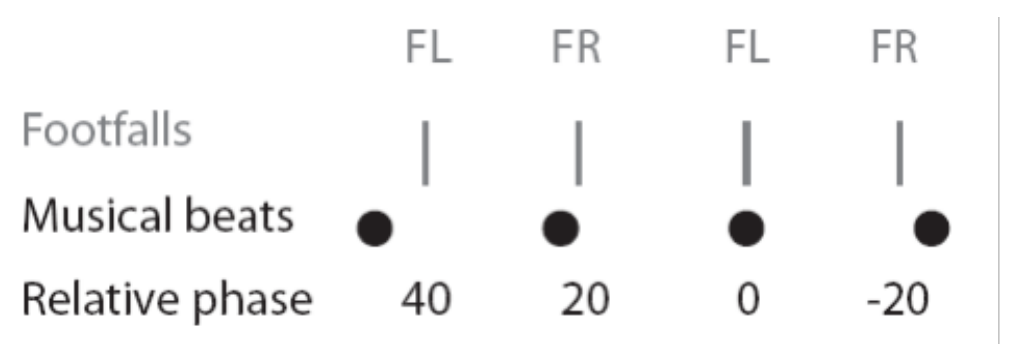

Fig. 3. Illustration of relative timing of footfalls and musical beats. Four footfalls (vertical grey lines) and four musical beats (black dots) are depicted $(\mathrm{FL}=$ Front left foot, $\mathrm{FR}=$ front right foot). The first two footfalls have positive relative phase (40 and 20 degrees) since they occur after the nearest musical beat. The third footfall has zero relative phase since it aligned in time with the musical beat. The fourth footfall has negative relative phase (-20 degrees) since it occurs before the nearest musical beat.

Once the RP sequence for a given trial was computed, we searched for bouts of synchrony by performing a windowed analysis on each sequence. Specifically, the first eight RP values were examined with a phasesensitive Rayleigh test (Fisher, 1993) to test if footfalls and beats were synchronized. Then, the window was shifted by 4 RP values and this test was repeated (i.e., window 1 was RP values 1-8, window 2 was RP values 5-12). This process of window-shifting and statistical testing was repeated until the end of the RP sequence was reached. A synchronized bout was defined as at least two consecutive windows (i.e., a minimum of 12 total RP values) with $p<0.05$.

How does the phase-sensitive Rayleigh test work? Briefly, this test uses a sequence of RP vectors to compute a mean vector, which can be represented on the unit circle (Figure 4). After computing this mean vector, the length of this resultant vector projected onto zero phase (the horizontal axis) provides a measure of synchrony. More intuitively, the test examines whether the RP vectors tend to cluster around zero phase, as would be expected if footfalls and beats were in synchrony and phase-matched.

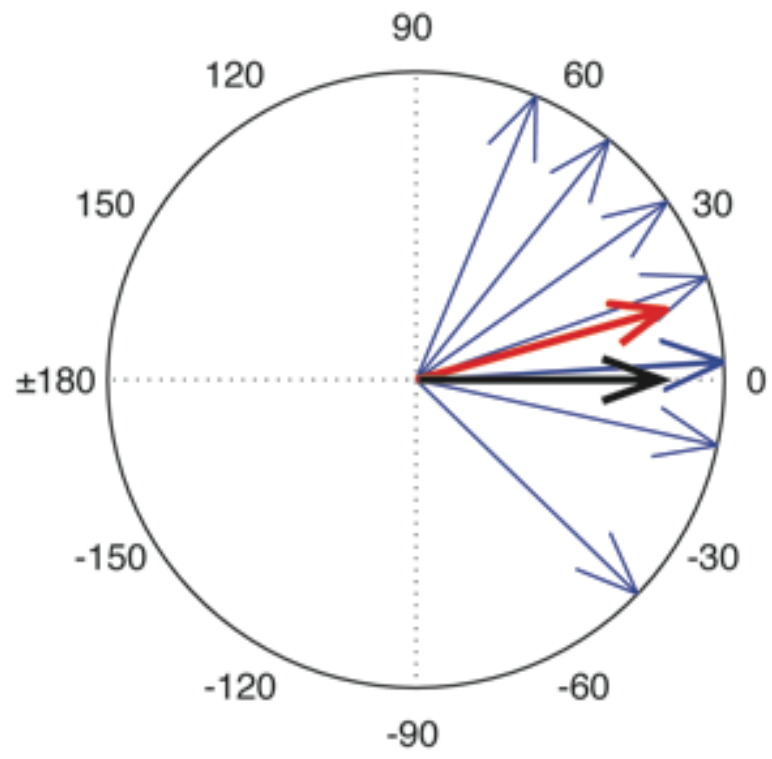

Fig. 4. Illustration of 8 relative phase values depicted as light blue vectors on the unit circle. The numbers around the circle are degrees ( 0 represents perfect alignment of footfall and musical beat; negative numbers indicate footfalls preceding the nearest musical beat; positive numbers indicate footfalls following the nearest beat). Note that two vectors have a very similar value (near 3 degrees) and hence overlap in this image. The dark red arrow is the mean vector of these eight phase vectors, and the black arrow is a projection of the mean phase vector onto the zero phase axis. 


\section{STATISTICAL SYNCHRONIZATION ANALYSIS: TESTING FOR BPS WITH A PERMUTATION TEST}

While the phase-sensitive Rayleigh test described above can identify synchronized bouts within each trial, one must consider the null hypothesis that such bouts occur by chance (cf. Patel et al., 2009b, subsection "Could synchronization have happened by chance?"). That is, one must consider the null hypothesis that the animal moves rhythmically in response to music, and that because of natural variability in movement tempo there are periods when (by pure chance) the movements have a consistent relationship to the beat. Patel et al. (2009a) used a permutation test to check this null hypothesis. The essential idea behind this test is to count the total number of movements (across trials) that are part of synchronized bouts, then to randomly pair each sequence of footfall timings with a musical beat time sequence from a different trial. The permuted data are then analyzed for synchronized bouts, and the total number of movements in such bouts is computed. If synchronized movements in the original data are largely due to chance, then there should be a comparable number of synchronized movements in the permuted data. To make this a valid statistical test, the process of permutation and synchronized-movement-counting must be done many times (e.g., 1,000), to create a distribution of values of the number of synchronized movements that are found by chance. The actual number of synchronized movements in the original data is then compared to this distribution to compute the probability (p-value) that the observed amount of synchrony is a chance result (for an application of this method to real data from another animal species, see Patel et al., 2009a).

For the permutation test to be meaningful, one must have trials at several different tempi, because this ensures variability in the structure of the musical beat time-series across trials. Since both of our musical stimuli were very close in tempo, and we were unable to conduct trials at different tempi, we could not apply the permutation test to our data. Future work with horses should endeavor to include at least five different tempi in the experiment, as noted previously in this paper. Synchronized bouts may not be found at all tempi, but if they are found at several tempi then the permutation test provides a way of testing whether the observed amount of synchrony is due to chance.

\section{Results from the current data}

We collected data from 16 trials and analyzed 15 of them (one trial was excluded since we were unable to get an accurate result from the beat-tracking algorithm). Trials varied in length from $21.5-62.7$ seconds (mean $=41.3$ seconds; trial onset was defined as the point when True started trotting to the music, and offset as the point when he ceased trotting, which always occurred before the music ended). The number of footfalls per trial ranged from 57-156 (mean $=101$ footfalls per trial, total number of footfalls across trials $=1537$ ). The two musical stimuli used for this experiment were very similar in tempo (both had tempi between 155.05 and 156.81 BPM when measured using the beat tracker).

After converting the footfall and musical beat timing data to sequences of relative phase values (as described above), we found that nearly all trials (14/15) had at least one synchronized bout (range: 1-4 bouts, mean: 1.79 bouts per trial). The number of footfalls that were part of synchronized bouts ranged from 12-72, with a mean of 35.42 synchronized footfalls per trial in the 14 trials with bouts (on these trials, an average of $33.9 \%$ of the footfalls were part of synchronized bouts). The total number of synchronized footfalls across all trials was 496. To test whether this amount of synchrony could occur by chance, and to test for tempo flexibility (a key feature of BPS), one would need data from trials at several different tempi, as discussed in the previous section.

Figure 5 shows relative phase data from one trial with a single bout of 40 synchronized footfalls. The synchronized bout is indicated with a black box. One notable aspect of this figure is that when True's tempo is slightly faster or slower than the music tempo, the relative phase slowly drifts from -180 to +180 degrees ("phase wrapping"), but during the bout stabilizes near zero, indicating footfalls at the same tempo as the music and synchronized with the musical beat.

In summary, True exhibits synchronized bouts, but given the limitations of this dataset it is not possible to say whether or not this is a coincidence. Testing genuine synchronization to a musical beat in horses awaits future research that includes more experimental trials and a range of musical tempi. 


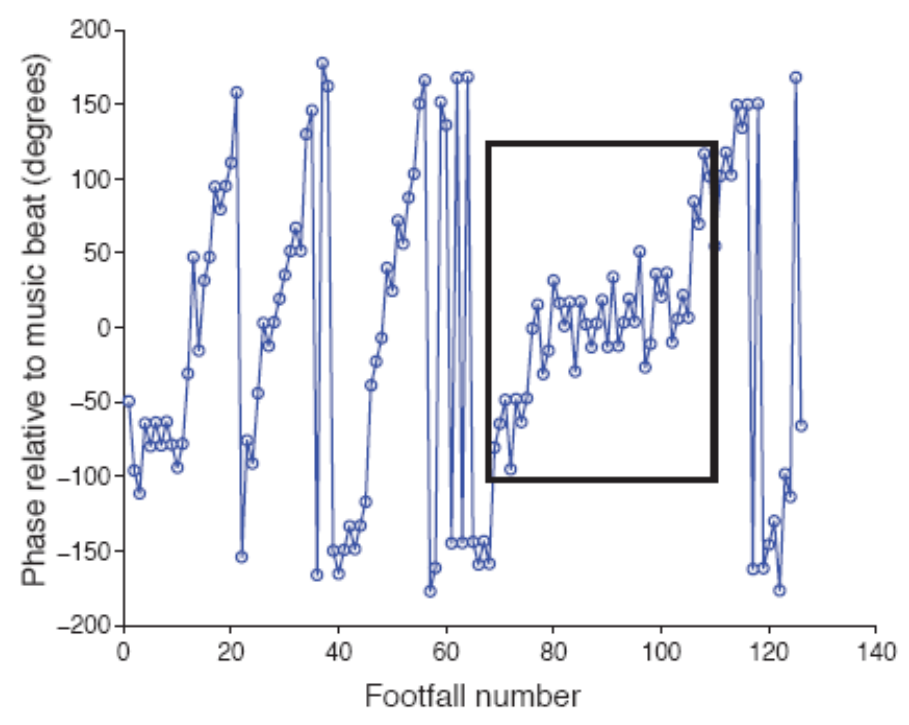

Fig. 5. Time series of relative phase values from one trial of the current study. The 40 values within the black box are part of a synchronized bout. Relative phase (in degrees) is plotted on the vertical axis with zero indicating perfect alignment of footfalls and musical beats. Negative values indicate footfalls that occur before the nearest beat, positive values indicate footfalls that occur after the nearest beat, and values of $+/-180$ indicate footfalls that are midway between beats (i.e., in antiphase with the beat).

\section{DISCUSSION}

We have presented a novel experimental method for testing synchronization to a musical beat in horses, and reviewed data analysis methods using pilot data collected from one horse. It is our hope that the methods presented here will be used to test the "vocal learning and rhythmic synchronization" hypothesis, which predicts that horses (and other vocal nonlearners) cannot synchronize their movements with a musical beat. Given current interest in human-horse interactions involving music (such as the Equus Projects, www.dancingwithhorses.org) and the scientific study of how animals process music (reviewed in Patel \& Demorest, 2013), hopefully such research will be conducted soon.

In closing, we briefly touch on a few conceptual issues relevant for future research on BPS in horses. The first is that there are over 300 horse breeds; the results obtained may depend on which breed is tested. Demonstration of BPS in even one breed would be sufficient to challenge the vocal learning hypothesis. The second issue concerns a conceptual distinction between tempo sensitivity and BPS. It may be that horses trot faster to fast music and slower to slow music, without showing true synchronization of movements to a musical beat. This would show tempo sensitivity, but not BPS. BPS involves the stable temporal alignment of rhythmic movements and musical beats, and further, is demonstrable at a range of different musical tempi (cf. Patel et al., 2009c). Finally, if after extensive testing it appears that horses are not capable of BPS, it would be worth asking if their lack of vocal-learning brain mechanisms is responsible, or if the key factor is the lack of some other ability also required for BPS. For example, the propensity to engage in joint social action (i.e., coordinated movement patterns with conspecifics) and the ability to imitate nonvocal movements may also be necessary foundations for BPS (Fitch, 2009; Patel et al., 2009b; Schachner, 2010). If horses have these two traits but are not capable of BPS, this would provide more specific support for the vocal learning hypothesis.

\section{ACKNOWLEDGMENTS}

We thank JoAnna Mendl Shaw of The Equus Projects for sharing her experience with horses, music, and dance with author AP and for introducing him to author DL. We also thank Sarah Gardner for her outstanding work as a research assistant, Sarah Gardner and Noah Friedman for coding True's movements from video, and Mark McLean for help with video recording. Supported by Neurosciences Research Foundation as part of its program on music and the brain at The Neurosciences Institute, where AP was the Esther J. Burnham Senior Fellow. 


\section{NOTES}

[1] Corresponding Author. Department of Psychology, Tufts University, 490 Boston Ave., Medford, MA, 02155. Email: a.patel@tufts.edu

\section{REFERENCES}

Arrighi, R., Alais, D., \& Burr, D. (2006). Perceptual synchrony of audiovisual streams for natural and artificial motion sequences. Journal of Vision, Vol. 6, pp. 260-268.

Bispham, J. (2006). Rhythm in music: What is it? Who has it? And why? Music Perception, Vol. 24, pp. 125-134.

Brown, S., \& Jordania, J. (2011). Universals in the world's musics. Psychology of Music, Advance online publication. DOI: 10.1177/0305735611425896

Buzsáki, G. (2006). Rhythms of the Brain. New York: Oxford University Press.

Darwin, C. (1871). The Descent of Man, and Selection in Relation to Sex. London: John Murray.

Ellis, D. (2007). Beat tracking by dynamic programming. Journal of New Music Research, Vol. 36, pp. 5160.

Fisher, N.I. (1993). Statistical Analysis of Circular Data. Cambridge: Cambridge University Press.

Fitch, W.T. (2006). The biology and evolution of music: A comparative perspective. Cognition, Vol. 100, pp. $173-215$.

Fitch, W.T. (2009). Biology of music: Another one bites the dust. Current Biology, Vol. 19, pp. R403-R404.

Gallo, T.C. (2007). You've got the beat. Practical Horseman, Vol. 35, pp. 47-49.

Georgopoulos, A.P., Taira, M., \& Lukashin, A. (1993). Cognitive neurophysiology of the motor cortex. Science, Vol. 260, pp. 47-52.

Gould, S.J. \& Vrba, C. (1982). Exaptation - a missing term in the science of form. Paleobiology, Vol. 8, pp. 4-15.

Hasegawa, A., Okanoya, K., Hasegawa, T., \& Seki, Y. (2011). Rhythmic synchronization tapping to an audio-visual metronome in budgerigars. Scientific Reports 1, 120; DOI:10.1038/srep00120.

Heffner, H.E., \& Heffner, R.S. (1992). Auditory perception. In: C. Phillips \& D. Piggins (Eds.), Farm Animals and the Environment. Wallingford, UK: C.A.B. Intl., pp. 159-184.

Honing, H., \& Ploeger, A. (2012). Cognition and the evolution of music: Pitfalls and prospects. Topics in Cognitive Science, Vol. 4, No. 4, pp. 513-524.

Houpt, K., Marrow, M., \& Seeliger, M. (2000). A preliminary study of the effect of music on equine behavior. Journal of Equine Veterinary Science, Vol. 20, No. 11, pp. 691-737.

Jarvis, E.D. (2007). Neural systems for vocal learning in birds and humans: a synopsis. Journal of Ornithology, Vol. 148 (Suppl. 1), pp. S35-S44.

Koelsch, S. (2011). Toward a neural basis of music perception - a review and updated model. Frontiers in Psychology, Vol. 2. 
McDermott, J.H., \& Hauser, M.D. (2005). The origins of music: Innateness, development, and evolution. Music Perception, Vol. 23, pp. 29-59.

McDermott, J.H., \& Hauser, M.D. (2007). Nonhuman primates prefer slow tempos but dislike music overall. Cognition, Vol. 104, pp. 654-668.

Nettl, B. (2000). An ethnomusicologist contemplates universals in musical sound and musical culture. In: N. L. Wallin, B. Merker, \& S. Brown (Eds.), The Origins of Music. Cambridge, MA: MIT Press, pp. 463472 .

Patel, A.D. (2006). Musical rhythm, linguistic rhythm, and human evolution. Music Perception, Vol. 24, pp. 99-104.

Patel, A.D., \& Demorest, S. (2013). Comparative music cognition: Cross-species and cross-cultural studies. In: D. Deutsch (Ed.). The Psychology of Music, $3^{\text {rd }}$ ed. San Diego: Academic Press/Elsevier, pp. 647-681.

Patel, A.D., Iversen, J.R., Bregman, M.R., \& Schulz, I. (2009a). Experimental evidence for synchronization to a musical beat in a nonhuman animal. Current Biology, Vol. 19, pp. 827-830.

Patel, A.D., Iversen, J.R. Bregman, M.R., \& Schulz, I. (2009b). Studying synchronization to a musical beat in nonhuman animals. Annals of the New York Academy of Sciences, Vol. 1169, pp. 459-469.

Patel, A.D., Iversen, J.R. Bregman, M.R., \& Schulz, I. (2009c). Avian and human movement to music: Two further parallels. Communicative and Integrative Biology, Vol. 2, pp. 1-4.

Patel, A.D., Iversen, J.R., Chen, Y.C., \& Repp, B.R. (2005). The influence of metricality and modality on synchronization with a beat. Experimental Brain Research, Vol. 163, pp. 226-238.

Peretz, I., \& Coltheart, M. (2003). Modularity of music processing. Nature Neuroscience, Vol. 6, pp. 688691.

Phillips-Silver, J., Toiviainen, P., Gosselin, N., Piché, O., Nozaradan, S., Palmer, C., \& Peretz, I. (2011). Born to dance but beat-deaf: A new form of congenital amusia. Neuropsychologia, Vol. 49, pp. 961-969.

Policht, R., Karadios, A., \& Frynta, D. (2011). Comparative analysis of long-range calls in equid stallions (Equidae): Are acoustic parameters related to social organization? African Zoology, Vol. 46, pp. 18-26.

Proops, L., Walton, M., \& McComb, K. (2010). The use of human-given cues by domestic horses, Equus caballus, during an object choice task. Animal Behaviour, Vol. 79, pp. 1205-1209.

Rankin, S.K, Large, E.W., \& Fink, P.W. (2009). Fractal tempo fluctuation and pulse prediction. Music Perception, Vol. 26, pp. 401-413.

Rauschecker, J.P., \& Scott, S.K. (2009). Maps and streams in the auditory cortex: Nonhuman primates illuminate human speech processing. Nature Neuroscience, Vol. 12, pp. 718-724.

Saslow, C.A. (2002). Understanding the perceptual world of horses. Applied Animal Behaviour Science, Vol. 78, p. 209-224.

Schachner, A. (2010). Auditory-motor entrainment in vocal-mimicking species: Additional ontogenetic and phylogenetic factors. Communicative \& Integrative Biology, Vol. 3, pp. 1-4.

Schachner, A., Brady, T.F., Pepperberg, I., \& Hauser, M. (2009). Spontaneous motor entrainment to music in multiple vocal mimicking species. Current Biology, Vol. 19, pp. 831-836.

Snowdon, C.T., \& Teie, D. (2010). Affective responses in tamarins elicited by species-specific music. Biology Letters, Vol. 6, pp. 30-32. 
Van Orden, K. (2005). Music, Discipline, and Arms in Early Modern France. Chicago: University of Chicago Press.

Wells, D. (2009). Sensory stimulation as environmental enrichment for captive animals: A review. Applied Animal Behaviour Science, Vol. 118, p. 1-11.

Wells, D.L., Graham, L., \& Hepper, P.G. (2002). The influence of auditory stimulation on the behavior of dogs housed in a rescue shelter. Animal Welfare, Vol. 11, pp. 385-393.

Wilson, E.O. (Ed.) (2006) From So Simple A Beginning: The Four Great Books of Charles Darwin. New York: W.W. Norton \& Co.

Zarco, W., Merchant, H., Prado, L., \& Mendez, J.C. (2009). Subsecond timing in primates: Comparison of interval production between human subjects and rhesus monkeys. Journal of Neurophysiology, Vol. 102, pp. 3191-3202.

\section{APPENDIX 1}

An anecdote about horse synchronization to a musical beat. The source is an Aug 11, 2009 essay "Using music to train your horse", from a blog about horse training (URL: http://www.carolynresnickblog.com/ using-music-to-train/). Bold font has been added to highlight critical sentences for this paper:

"Over the years, I have discovered that horses not only listen to music, they respond to music with rhythmic strides. I also found the music increases their desire to work close with me in the training process.

At the age of 10, I began training horses for the show arena. I had also studied ballet most of my childhood and planned to be a professional ballet dancer. However, when I reached the age of 18, I had to give up dancing due to an inner ear problem that affected my balance. It was the natural choice to pursue a career in showing horses. I fulfilled my need for dancing by listening to music when I was around the barn. If you should drop by my ranch, you would hear anything from Swan Lake to country music. It wasn't until years later that I started playing music while I trained horses.

One day I was riding and listening to music when I noticed the horse I was riding was keeping tempo to the music. It was uncanny. I wasn't sure if my listening to music was influencing the manner in which I gave the signals that I communicated to my horse or if he was choosing to let the music influence his steps. Perhaps it was a little of both. Whatever it was, I this found unity we were sharing. Then I noticed horse after horse that I rode was responding the same way to the music. I became more creative in my training sessions asking the horse to perform the rhythm or various types of music. What I discovered when I took this approach surprised me.

The horse learned their new elements much faster. They seemed to understand my need to stay within the tempo. It was a marvelous discovery. I experimented and turned the horses loose on their own to see how much I had influenced them. What I learned was amazing. On their own, each horse stepped to the music perfectly like a metronome. Horse after horse, at liberty, ran, walked and trotted in time with the music. The horses demonstrated that they were actively listening to the music and being attentive to the tempo and rhythm."

\section{APPENDIX 2}

Videos 1-6:

http://library.osu.edu/ojs/public/journals/8/EMR v7 03-04 video1-6.html

Videos 7-12:

http://library.osu.edu/ojs/public/journals/8/EMR v7 03-04 video7-12.html

Videos 13-16:

http://library.osu.edu/ojs/public/journals/8/EMR_v7_03-04_video13-16.html 